\title{
Triceps Brachialis response of Radial Nerve assessed with neurostimulation during axillary plexus blockade: clinical, anatomical and histological correlation.
}

\author{
A.Martínez-Camacho1, V.Valera11, C. Ruiz¹, A. Ruiz¹, J.A.Garcia1', X. Sala-Blanch¹,2 \\ 1. Servei d' Anestesiologia i Reanimació- Hospital Clínic de Barcelona \\ 2. Universitat de Barcelona
}

Introduction: Axillary plexus blockade is a common technique in clinical practice with the pattern of structures around the artery well known. Historically, the proper response for radial nerve stimulation was only considered the extension of the hand and wrist.

Materials and Methods: Twenty-five axillary blockades assessed by ultrasound and neurostimulation; the principal objective was to correlate the needle position over the nerve and correlate the findings with the anatomical and histological structure of the radial nerve. During the procedure, the neurostimulation needle was directed in two directions: above the axillary artery or underneath it to reach the medial or lateral margin of the nerve. Once the needle reached the nerve, the current was augmented gradually until a response was elicited. For the anatomical analysis, histological samples and eight and axillas were dissected to correlate the results with the anatomy.

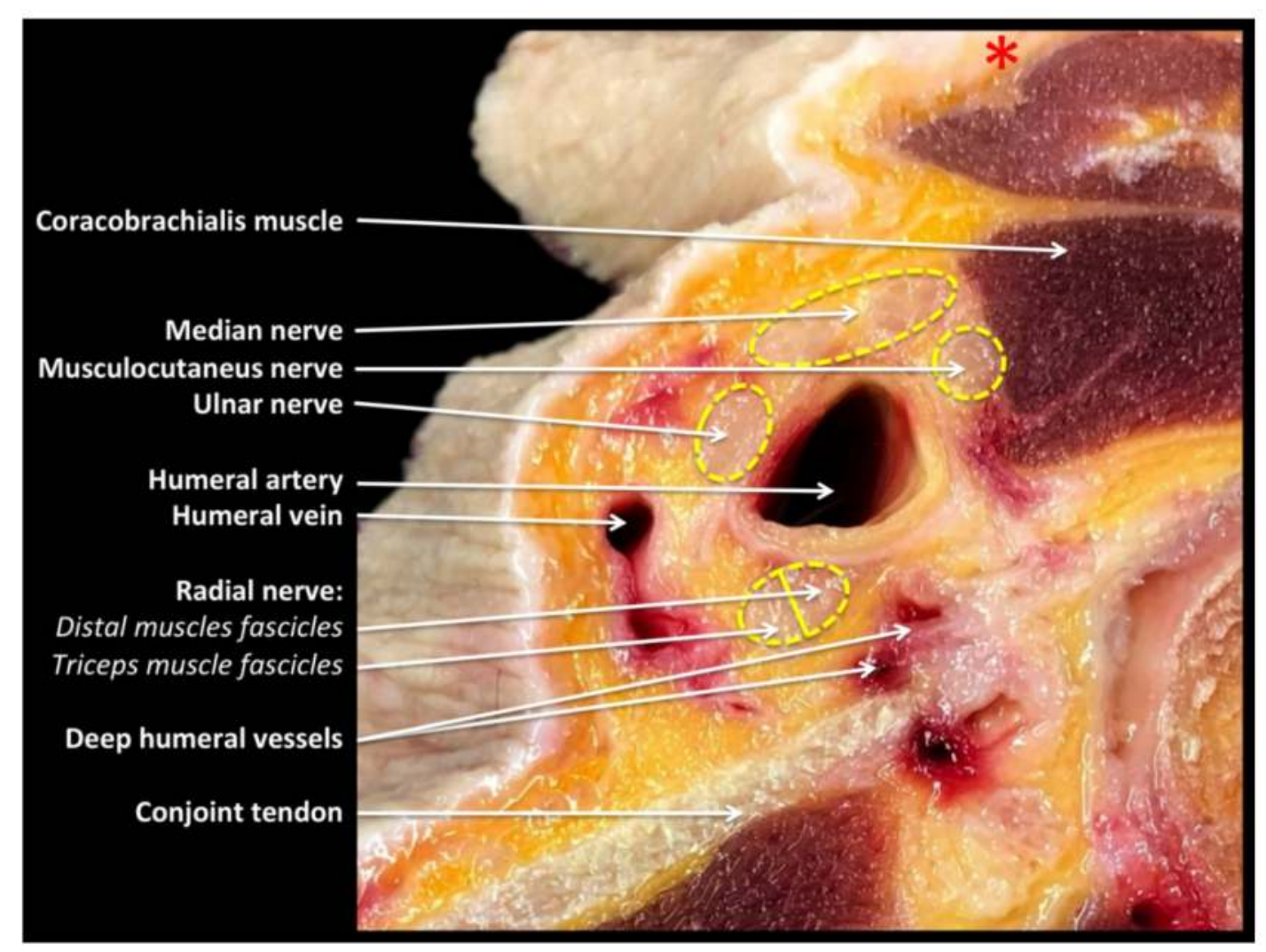

Fig.1. Cross-section of the arm obliquely through the latissimus dorsi tendon to the insertion of the pectoralis major on the humerus.

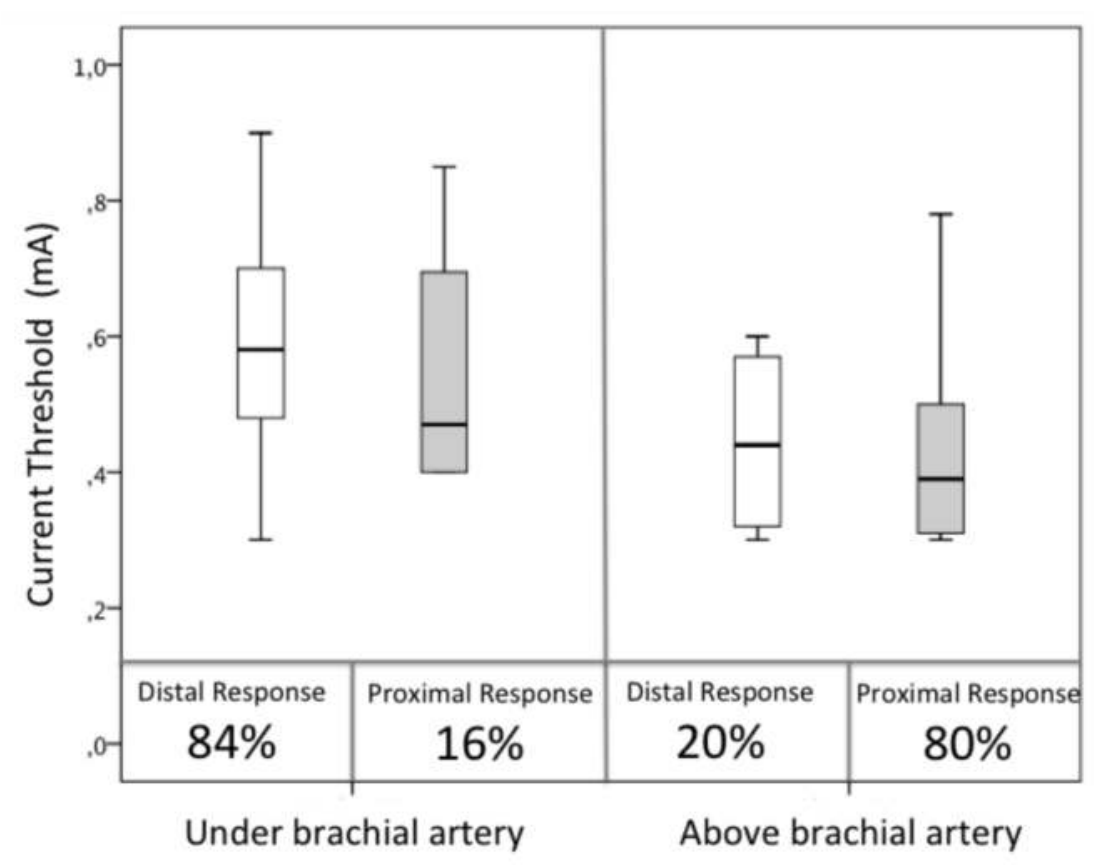

Fig.3. Minimal current (mA) that causes motor responses to the course of the needle, above or below the artery, to the percentages of proximal or distal motor response
Results: The results obtained, showed a significant difference in the relationship with the pathway of the needle with the artery and the motor response evoked $(p<0,001)$ and mean intensity of stimulation is significantly lower when the access to the nerve is performed above the artery $(0,44 \pm 0,15 \mathrm{~mA})$ in contrast to the access under it $(0,57 \pm 0,17 \mathrm{~mA})(p=0,015)$.

Conclusion: a triceps brachialis motor response once the radial nerve is reached above the artery and over the conjoint tendon, occurs at lower current intensities and therefore distance needle-nerve. Thus, is an adequate response considering the location of the radial nerve and the neural topography at this situation.
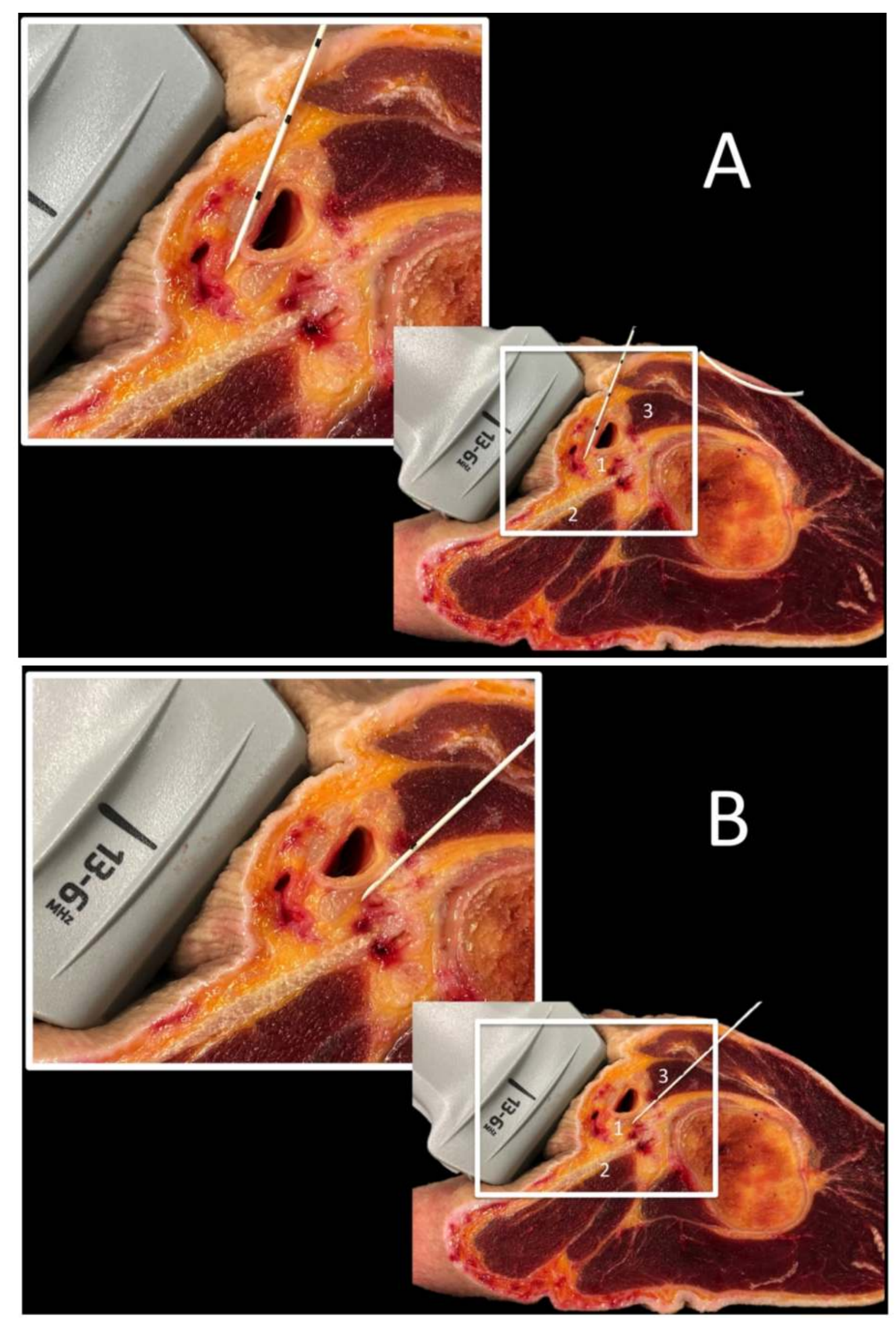

Figure 2. Anatomical dissection showing the location of the neurostimulation needle during its approach to the radial nerve from both directions: $(A)$ under the artery; $(B)$ over the artery. 